\title{
Topology Design for Distributed Formation Control towards Optimal Convergence Rate
}

\author{
Huang Huang, Changbin Yu, Azwirman Gusrialdi and Sandra Hirche
}

\begin{abstract}
This paper considers the optimal local leader selection for a leader-first follower minimally persistent formation system. The objective is to maximize the local convergence rate of the followers to the unique equilibrium under small perturbations. Except for the leader and the first follower, every other agent in the system follows exactly two agents called the local leaders and is responsible for maintaining a pair of desired distances from two local leaders. The control algorithm is the linearized form of the decentralized nonlinear control law proposed in our previous work. When the agents are distributed over a rectangular area, the selection of the optimal local leader for each follower is discussed, and it is discovered that the boundary optimality rule applies. The general case when agents distributed over an arbitrary convex domain is further considered based on the matrix perturbation theory. Information of agents in its sensing range is enough for the agent to pick up its optimal local leaders, which allows a distributed implementation of the proposed algorithm.
\end{abstract}

\section{INTRODUCTION}

It is generally recognized that the underlying topology (or the communication topology) of a formation system is closely related to the stability as well as cooperative performance of the system. In the absence of communication cost, a dense network topology is always beneficial to the convergence rate of the formation, as proved in [1]. Also the design of the underlying topology should avoid containing cyclic subgraph as it reduces the robustness of the system [2]. A stiffness matrix and the worst case rigidity index were proposed in [3] to measure the efforts required for the deformation of a rigid formation system. Following this work, Kim et al. [4] further proposed an iterative algorithm, which was a centralized one, that reconfigured the positions and the connection topology so as to optimize the rigid graph. The problem of single leader selection was discussed in [5] based on the information each agent observed. Similar discussions on topology optimization are seen in consensus algorithms as well. In order to accelerate the convergence rate, Kar et. al [6] proved that a special class of regular graphs, viz., the

H. Huang is with Beijing Institute of Control Engineering, and is also with Science and Technology on Space Intelligent Control Laboratory, Beijing, China. This work was completed during H. Huang's visit at the Australian National University and was supported by the National Natural Science Foundation of China under grant No. 60736023 and grant No. 60704014. hhuang33@gmail. com

C. Yu is with the Australian National University, supported by the Australian Research Council through Queen Elizabeth II Fellowship under DP-110100538. He is also with Shandong Computer Science Center, supported by the overseas expert program of Shandong Province. brad.yulanu.edu. au

A. Gusrialdi and S. Hirche are with Institute of Automatic Control Engineering, Technische Universität of München, München, D-80290 München, Germany
Ramanujan graphs, has the maximal algebraic connectivity. In the highly complex small-world network, by rewriting edges of $G^{k}$ when $G$ being a ring graph at probability $p$ between 0.01 and 1 , the algebraic connectivity of the graph increases dramatically, and hence the convergence rate, as $p$ approaches 1 [7]. Recently, leader selection for leaderfollower consensus algorithm was discussed in [8] from the $H_{2}$ norm perspective.

In this paper, we consider formation control over a minimally persistent graph. For each pair of agents adjacent to one edge, only one of them is assigned to maintain a desired distance. In order to generate the optimal underlying graph autonomously and in a distributed way, we consider subsequential steps where at each step, only one unsettled agent, i.e., agent whose outgoing edges are unknown, is considered. Two local leaders to which this unsettled agent will follow are picked up at each step without changing the existing edges. Starting from an existing graph that is minimally persistent, this sequential procedure guarantees the minimal persistency of the new graph at each step [9]. Although there are other ways to construct a minimally persistent graph as discussed in [9], the procedure we consider is implemented in a distributed way. The optimality is evaluated by the local convergence rate of each agent, i.e., the rate of the convergence of each agent towards its desired position. We only consider systems subject to small perturbations such as temporal communication delay, uneven surface or wind gust. A large local convergence rate allows disturbed agents to regain the assigned formation fast and thus is favorable to the mission execution time.

In Section II, the linearized dynamics and the decentralized control law are introduced. The situations with candidate local leaders densely distributed over a rectangular area and an arbitrary convex hull are discussed in Section III and Section IV respectively. Finally Section V gives the conclusion.

\section{Problem Setup}

Let $\mathbb{R}\left(\mathbb{R}^{+}\right)$be the set of (positive) real numbers and $\mathbb{R}^{m \times n}$ be the set of real matrices of size $m \times n$. With $n=1, \mathbb{R}^{m \times n}=\mathbb{R}^{m}$. The set of eigenvalues of a matrix $M \in \mathbb{R}^{m \times m}$ is denoted by $\lambda(M)$. The spectral abscissa of $M$, denoted by $\alpha(M)$, is the largest real part of all the eigenvalues. $M^{T}$ is the transpose of matrix $M$. The real inner product of two real square matrices is:

$$
\langle A, B\rangle=\operatorname{tr} A^{T} B=\sum_{i, j} a_{i j} b_{i j}
$$


For a complex vector $u$ of dimension $n \times 1$, its conjugate transpose is $u^{*}$, and its real part and imaginary part are denoted by $\operatorname{Re}(u) \in \mathbb{R}^{n}$ and $\operatorname{Im}(u) \in \mathbb{R}^{n}$ respectively.

A directed graph $G=(V, E)$ has $|V|$ vertices and $|E|$ directed edges. For simplicity, a graph with $N$ nodes is usually denoted by $G_{N}$. The underlying graph of a formation system is determined in a way that if agent $i$ is responsible for keeping the distance from agent $j$, then agent $j$ is the local leader of agent $i$ and there is a directed edge $e_{i j} \in E$ with the tail at agent $i$ and the head at agent $j$. The group leader in a formation system is the one with no outgoing edges in the underlying graph and the first follower follows only the group leader. For a formation system consists of $N$ agents, the group leader is marked agent $N$ and the first follower is marked agent $N-1$.

A formation system with a minimally persistent graph is called a minimally persistent formation system. If there is only one group leader and one first follower in the graph, it is further called a leader-first follower minimally persistent (LFFMP) formation system. Decentralized nonlinear control laws for the LFFMP system were proposed in our previous work [10] that restored the desired formation in the presence of small distortions from the nominal one. The system model and the control law in [10] are borrowed in this paper and thus are restated below.

Assume the displacements brought by the small perturbations are small enough to allow first order approximation. In particular the position of agent $i$, denoted by $p_{i}(t)=$ $\left[\begin{array}{ll}x_{i}(t) & y_{i}(t)\end{array}\right]^{T}$, can be represented at all times by $p_{i}(t)=$ $\delta p_{i}(t)+\bar{p}_{i}$, where $\bar{p}_{i}=\left[\bar{x}_{i} \bar{y}_{i}\right]^{T}$ corresponds to agent $i$ 's position for which all desired local distance constraints are met at time instance $t$, and $\delta p_{i}(t)=\left[\delta x_{i}(t) \delta y_{i}(t)\right]^{T}$ is small, see Fig. 1. Then the linearized dynamics of follower $i \in[1, N-2]$ that has two outgoing edges are

$$
\begin{gathered}
{\left[\begin{array}{c}
\delta \dot{x}_{i} \\
\delta \dot{y}_{i}
\end{array}\right]=K_{i}\left[\begin{array}{ll}
-\delta \bar{x}_{i, j} & -\delta \bar{y}_{i, j} \\
-\delta \bar{x}_{i, k} & -\delta \bar{y}_{i, k}
\end{array}\right]^{-1} R_{(i j, i k)}\left[\begin{array}{l}
\delta x_{i} \\
\delta y_{i} \\
\delta x_{j} \\
\delta y_{j} \\
\delta x_{k} \\
\delta y_{k}
\end{array}\right]} \\
\triangleq K_{i} R_{e_{i}}^{-1} R_{(i j, i k)}\left[\begin{array}{lll}
\delta p_{i} & \delta p_{j} & \delta p_{k}
\end{array}\right]^{T}
\end{gathered}
$$

with

$R_{(i j, i k)}=\left[\begin{array}{cccccc}\delta \bar{x}_{i, j} & \delta \bar{y}_{i, j} & -\delta \bar{x}_{i, j} & -\delta \bar{y}_{i, j} & 0 & 0 \\ \delta \bar{x}_{i, k} & \delta \bar{y}_{i, k} & 0 & 0 & -\delta \bar{x}_{i, k} & -\delta \bar{y}_{i, k}\end{array}\right]$ and $\delta \bar{x}_{i, j}=\bar{x}_{i}-\bar{x}_{j}, \delta \bar{y}_{i, j}=\bar{y}_{i}-\bar{y}_{j}$. Matrix $K_{i}$ is the feed forward control gain to be designed.

Due to the fact that the first follower moves solely along a line directed to the leader, the dynamics for the first follower agent $N-1$ are

$$
\left[\begin{array}{l}
\delta \dot{x}_{N-1} \\
\delta \dot{y}_{N-1}
\end{array}\right]=\left[\begin{array}{c}
-k_{N-1} \delta x_{N-1} \\
0
\end{array}\right]
$$

and for the group leader, we assume it does not move (in this paper it is assumed that each agent knows the velocity of the leader such that the stillness of the leader holds). In

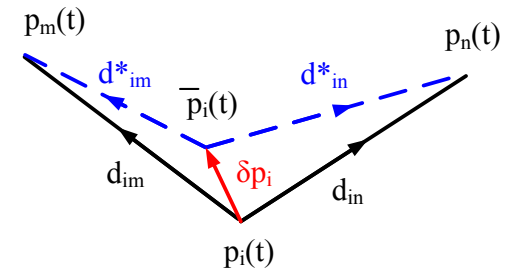

Fig. 1: Illustration of position adjustment for point agent $i$ at $p_{i}$ with respect to agent $m$ at $p_{m}$ and agent $n$ at $p_{n}$. The desired position is at $\bar{p}_{i}$

the light of the above discussion, the overall dynamics of the LFFMP formation system is

$$
\delta \dot{\hat{p}}(t)=\hat{K} \hat{R}^{-1} \hat{R} \delta \hat{p}(t): \triangleq A_{c} \delta \hat{p}(t)
$$

where $\hat{K}=\operatorname{diag}\left[K_{1}, K_{2}, \ldots, K_{N-2}, k_{N-1}\right]$, $\hat{R}_{e}=\operatorname{diag}\left[R_{e_{1}}, \ldots, R_{e_{N-2}}, \delta \bar{x}_{N, N-1}\right]$ and $\delta \hat{p}=$ $\left[\delta p_{1}, \ldots, \delta p_{N-2}, \delta x_{N-1}\right] . \quad \hat{R}$ is obtained by removing the last three columns from the rigidity matrix $R$ of the minimally persistent graph.

Lemma 2.1: There exists a diagonal matrix $\Lambda \in$ $\mathbb{R}^{(2 N-3) \times(2 N-3)}$ such that $\Lambda \hat{R}$ has all eigenvalues in the open left half plane and by choosing

$$
\hat{K}=\Lambda \hat{R}_{e}
$$

the formation system with the feedforward controller $\hat{K}$ is stable on its equilibrium.

This is a corollary of Theorem 3.2 and Theorem 4.1 in [10]. Under control law (4), the formation system (3) is then

$$
\delta \dot{\hat{p}}(t)=\Lambda \hat{R} \delta \hat{p}(t) .
$$

Assume there are $N+M$ agents in the desired formation where all the desired interagent distances are satisfied. The $N$ agents, labeled from 1 to $N$, are connected over an assigned and fixed minimally persistent graph $G_{N}$ without any outgoing edges to the other $M$ agents labeled from $N+1$ to $N+M$. The $M$ agents are the unsettled agents with unknown local leaders. Each of these agents has to choose two local leaders to follow from their own sensing ranges $S_{i} \in \mathbb{R}^{+}$. For example in Fig. 2, agent $N+1$ to $N+4$ are unsettled agents and the goal is to determine the pair of local leaders for $N+i, i \in[1,4]$ so that the convergence rate of agent $N+i$ to its equilibrium is maximized under small perturbations.

The design of the optimal minimally persistent graph is a combinatorial optimization problem. We break down the problem into $M$ subsequential steps. At step $i$, the two local leaders for agent $N+i$ are picked up from the set of candidate local leaders (CLL), denoted by $C_{N+i}=\left\{k \mid\left\|p_{k}-p_{N+i}\right\| \leq\right.$ $\left.S_{N+i}\right\}$, without changing the underling graph of the previous $N+i-1$ agents. As long as $G_{N+i-1}$ is minimally persistent, the proposed sequential steps will consistently produce a minimally persistent graph $G_{N+i}$ for the $N+i$ agents [9]. Meanwhile, for the $N+i-1$ agents over a minimally persistent graph, by reordering the $N+i-1$ agents, their 


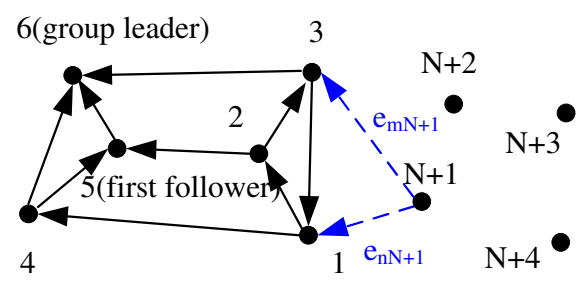

Fig. 2: Adding an unsettled agent to the existing minimally persistent graph. Agent 6 is the group leader and agent 5 is the first follower.

linearized dynamics follow the one given in (3). When agent $N+i$ joins and takes agent $m$ and agent $n$ as its local leaders, the system matrix of the $N+i$ agents is then

$$
\tilde{A}_{c}=\left[\begin{array}{cc}
A_{c} & 0 \\
Q_{N+i} & K_{N+i}
\end{array}\right]
$$

where $A_{c}$ given in (3) is the dynamics of the $N+i-1$ agents and according to (4), matrix $K_{N+i}$ is given by

$$
K_{N+i}=\left[\begin{array}{cc}
k_{1} & 0 \\
0 & k_{2}
\end{array}\right]\left[\begin{array}{ll}
\bar{x}_{N+i}-\bar{x}_{m} & \bar{y}_{N+i}-\bar{y}_{m} \\
\bar{x}_{N+i}-\bar{x}_{n} & \bar{y}_{N+i}-\bar{y}_{n}
\end{array}\right] .
$$

and

$$
\begin{aligned}
& Q_{N+i}=\left[\begin{array}{lllllll}
0 & \cdots & Q_{N+i}^{(m)} & \cdots & Q_{N+i}^{(n)} & \cdots & 0
\end{array}\right] \\
& Q_{N+i}^{(m)}=k_{1}\left[\begin{array}{cc}
-\bar{x}_{N+i}+\bar{x}_{m} & -\bar{y}_{N+i}+\bar{y}_{m} \\
0 & 0
\end{array}\right] \\
& Q_{N+i}^{(n)}=k_{2}\left[\begin{array}{cc}
0 & 0 \\
-\bar{x}_{N+i}+\bar{x}_{n} & -\bar{y}_{N+i}+\bar{y}_{n}
\end{array}\right]
\end{aligned}
$$

$k_{1}$ and $k_{2}$ are the scaling parameters. In this paper in order to focus on the topology design issue, we only consider cases when $k_{1}=-1$ and $k_{2}=1$.

Problem 2.1: Consider a formation system consists of $N$ agents labeled from 1 to $N$ over a fixed minimally persistent graph and $M$ unsettled agents labeled from $N+1$ to $N+M$. According to the relative distances constraints, each agent calculates its desired position. Find the optimal two local leaders $m$ and $n$ for unsettled agent $i$ such that

$$
\begin{array}{ll}
\min _{m, n} & \alpha\left(K_{N+i}\right) \\
\text { s.t. } & m, n \in C_{N+i},
\end{array}
$$

Eigenvalues of a matrix are highly sensitive to perturbations on the entries. In fact, they are non-Lipschitz. Although the optimization of the spectral abscissa of a symmetric matrix is convex, for the nonsymmetric case, it is nonconvex and nonsmooth. Thus finding global optimum of the spectral abscissa over a stable nonsymmetric matrix is hard. Burke et. al [11] proposed a numerical method based on random gradient bundle method for the approximating local minimizers. This optimization technique only applies to problems over a continuous domain, and thus is not applicable directly to Problem 2.1.

In this paper, we first solve the problem over a continuous domain to obtain the optimal solutions. By referring to these optimal solutions, the actual optimal pair of local leaders is then selected from the discrete set $C L L_{i}$ based on the gradient method and the matrix perturbation theory. Thus it is assumed that

Assumption 2.1: All the agents are densely distributed over an area such that for any optimal solutions obtained on the continuous domain, there is always at least one pair of agents located nearby, which allows at least one pair of agents whose $\alpha\left(K_{N+i}\right)$ is on the same convex surface as that of the local minimizer.

\section{CLLS DISTRIBUTED OVER A RECTANGLE DOMAIN IN QUADRANT I}

In this section, we consider a simple and special situation: the candidate local leaders of agent $N+i$ are densely distributed over a rectangle such that

Assumption 3.1: By centering the reference frame at $\bar{p}_{N+i}$, there is a minimal rectangle that covers the CLLs and its sides are parallel to the $\mathrm{x}$-axis and $\mathrm{y}$-axis. Denote the rectangle by $(\underline{x}, \bar{x}, \underline{y}, \bar{y})$ where $(\underline{x}, \underline{y})$ and $(\bar{x}, \bar{y})$ are the coordinates of its bottom left corner and its upper right corner respectively.

As will be showed later, a rectangle distribution allows easy analysis on the optimal solution because of the invariance of $\mathrm{x}$ or $\mathrm{y}$ along the edges. The more general case will be discussed later in the nex section.

Based on the relative position of the rectangle and the $x-y$ axis, the positions of the rectangle in the new reference frame have eight possible cases. For instance, the rectangle could locate either in quadrant I or span over quadrant I and quadrant IV. Note that the case when the rectangle covers the origin is excluded because at the current stage, we only consider new agents joining the group from outside.

Under Assumption 3.1, variables $\bar{x}_{m}$ and $\bar{x}_{n}$ are decoupled from $\bar{y}_{m}$ and $\bar{y}_{n}$ respectively. As indicated in [11], the optimal solution is obtained when the two eigenvalues of $K_{N+i}$ are as coalesce to each other as possible, i.e.,

$$
\bar{x}_{m}^{2}+\bar{y}_{n}^{2}+2 \bar{x}_{m} \bar{y}_{n}-4 \bar{y}_{m} \bar{x}_{n} \leq 0
$$

is feasible, the optimal spectral abscissa is $\alpha\left(K_{N+i}\right)=$ $1 / 2\left(\bar{x}_{m}-\bar{y}_{n}\right)$.

Theorem 3.1: Let the minimal rectangle in Assumption 3.1 be located in quadrant I. When $\bar{y}>\underline{x}$, the optimal solution over the continuous rectangle domain exhibits the following boundary optimality:

1) The optimal solutions consist of points on the left edge and the top edge respectively if

$$
\bar{x}>\frac{(\underline{x}+\bar{y})^{2}}{4 \bar{y}}
$$

and the optimal solutions are such that

$$
4 \bar{y}_{m} \bar{x}_{n} \geq(\underline{x}+\bar{y})^{2}
$$

with the optimal spectral abscissa $\alpha^{\star}\left(K_{N+i}\right)=\underline{x}-\bar{y}$. 

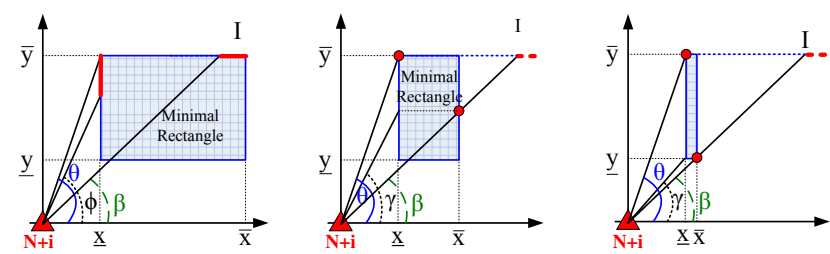

(a) Optimal pair of local (b) Only one optimal so- (c) Only one optimal soleaders lie in the two red lution bold lines respectively lution

Fig. 3: Optimal solutions when the rectangle is on quadrant I. When $\bar{x}$ decreases, the optimal pair of solutions appear in different positions.

2) The only optimal solution consists of points on the upper left corner and the right edge respectively if

$$
\frac{(\underline{x}+\underline{y})^{2}}{4 \bar{y}}<\bar{x}<\frac{(\underline{x}+\bar{y})^{2}}{4 \bar{y}}
$$

and the optimal spectral abscissa $\alpha^{\star}\left(K_{N+i}\right)=\underline{x}-\bar{y}_{n}^{\star}$ with $\bar{y}_{n}^{\star}=\sqrt{4 \bar{y} \bar{x}}-\underline{x}$.

3) The only optimal solution consists of points on the upper left corner and the lower right corner respectively if

$$
\bar{x}<\frac{(\underline{x}+\underline{y})^{2}}{4 \bar{y}}
$$

with the optimal spectral abscissa $\alpha^{\star}\left(K_{N+i}\right)=\underline{x}-$ $\underline{y}+\sqrt{(\underline{x}+\underline{y})^{2}-4 \bar{y} \bar{x}}$

Proof: The minimum of $\alpha\left(K_{N+i}\right)=1 / 2\left(\bar{x}_{m}-\bar{y}_{n}\right)$ is when $\bar{x}_{m}=\underline{x}$ and $\bar{y}_{n}=\bar{y}$ if there exist $\bar{y}_{m}$ and $\bar{x}_{n}$ such that condition (6) is satisfied. Thus condition (7) is concluded. Similar proofs apply to 2) and 3) as well. Fig. 3(a) further gives an illustration of the three situations.

The three situations in Theorem 3.1 are distinguished by the upper bound of $\bar{x}$. When $\bar{x}$ decreases, the positions of the optimal solutions change accordingly, as in Fig. 3 .

Remark 3.1: Let $\phi, \beta$ and $\theta$ be the angles defined in Fig. 3(a). $\phi$ and $\beta$ restricted the area where the optimal solutions appear. The optimal solutions to situation 1) are subject to the angle constraints of

$$
\tan \phi \geq \frac{1}{2} \tan \beta\left(1+\frac{1}{\sin 2 \theta}\right)
$$

As $\theta>\pi / 4$, the lower bound of $\tan \phi$ is positive monotonic to $\beta$.

Remark 3.2: Let $\gamma, \beta$ and $\theta$ be the angles defined in Fig. 3 (b). The angle constrains for situation 2) is

$$
\tan \beta=\frac{2 \tan \theta \sin 2 \gamma}{1+\sin 2 \gamma}
$$

When $\bar{x}$ decreases, $\bar{y}_{n}^{\star}$ decreases as well and the extreme situation tends to be the one in Fig. 3(c). After the optimal solution reaches the corners, it stays there irrespective of the decrease of $\bar{x}$.

As can be observed, the locations of the optimal solutions are closely related to the shape and the position of the rectangle.
Now we are ready to present the solution to Problem 2.1. The control gain $K_{N+i}$ is an affine function

$$
\begin{aligned}
& K_{N+i} \\
& =\bar{x}_{m}\left[\begin{array}{ll}
1 & 0 \\
0 & 0
\end{array}\right]+\bar{y}_{m}\left[\begin{array}{ll}
0 & 1 \\
0 & 0
\end{array}\right]+\bar{x}_{n}\left[\begin{array}{cc}
0 & 0 \\
-1 & 0
\end{array}\right]+\bar{y}_{n}\left[\begin{array}{cc}
0 & 0 \\
0 & -1
\end{array}\right] \\
& \triangleq \bar{x}_{m} K_{1}+\bar{y}_{m} K_{2}+\bar{x}_{n} K_{3}+\bar{y}_{n} K_{4}
\end{aligned}
$$

and it is well known [12] that when $K_{N+i}$ has a conjugate pair of eigenvalues, its gradient is given by

$$
\left(\nabla \alpha\left(K_{N+i}\right)\right)_{k}=\mathbf{R e}(u)^{T} K_{k} \mathbf{R e}(v)+\mathbf{I m}(u)^{T} K_{k} \mathbf{I m}(v)
$$

where $v$ and $u$ are the right and left eigenvectors corresponding to either of the complex eigenvalues (but the same one) of $K_{N+i}$ and satisfy $u^{*} v=1$.

Under small perturbations, the variation of $\alpha\left(K_{N+i}\right)$ is

$$
\Delta \alpha\left(K_{N+i}\right)=\left\langle\nabla\left(\alpha\left(K_{N+i}\right)\right), \Delta K_{N+i}\right\rangle
$$

Theorem 3.2: For the optimal solutions in situation 1) in Theorem 3.1, the gradient on agent $m$ is perpendicular to the left side and the one on agent $n$ is perpendicular to the top side.

Proof: The equality of two complex numbers means the equality of their real part and imaginary part respectively, which yields,

$$
M \operatorname{Re}(v)=-\mathbf{I m}(\lambda) \operatorname{Im}(v) \quad M^{T} \mathbf{I m}(u)=\mathbf{I m} \lambda \mathbf{I m}(u)
$$

where $M=K_{N+i}-\boldsymbol{R e}(\lambda) I$.

Due to $u^{*} v=1$, we have

$$
\boldsymbol{\operatorname { R e }}(u)^{T} \mathbf{I m}(v)=\mathbf{I m}(u)^{T} \operatorname{Re}(v)
$$

Substituting (14) into (13), we have

$$
\boldsymbol{\operatorname { R e }} u^{T} M \mathbf{R e} v+\mathbf{I m} u^{T} M \mathbf{I m} v=0
$$

It has been proved that $\operatorname{Re}(\lambda)=1 / 2\left(\bar{x}_{m}-\bar{y}_{n}\right)$. Referring to (12), this equation has the explicit form of

$$
\frac{\bar{x}_{m}+\bar{y}_{n}}{2}\left(\nabla \phi_{1}+\nabla \phi_{4}\right)+\bar{y}_{m} \nabla \phi_{2}-\bar{x}_{n} \nabla \phi_{3}=0
$$

where $\nabla \phi_{i}=\left(\nabla \alpha\left(K_{N+i}\right)\right)_{i}$ and $\left(\nabla \alpha\left(K_{N+i}\right)\right)_{1}-$ $\left(\nabla \alpha\left(K_{N+i}\right)\right)_{4}=\operatorname{Re}(u)^{T} \operatorname{Re}(v)+\operatorname{Im}(u)^{T} \mathbf{I m}(v)^{T}=$ 1. According to the positiveness of the corresponding variables, the necessary and sufficient condition for eq. (15) is $\left.\left(\nabla \alpha\left(K_{N+i}\right)\right)_{1}=-\left(\nabla \alpha\left(K_{N+i}\right)\right)_{4}\right)=0.5$ and $\left(\nabla \alpha\left(K_{N+i}\right)\right)_{k}=0, k=2,3$.

The optimal solution corresponds to two points in the plane, and consequently the gradient could also be divided into two vectors in the plane, i.e.,

$$
v_{1}=\left[\begin{array}{c}
\nabla \phi_{1} \\
\nabla \phi_{2}
\end{array}\right]=\left[\begin{array}{c}
0.5 \\
0
\end{array}\right], \quad v_{2}=\left[\begin{array}{c}
\nabla \phi_{3} \\
\nabla \phi_{4}
\end{array}\right]=\left[\begin{array}{c}
0 \\
-0.5
\end{array}\right]
$$

which finishes the proof.

Remark 3.3: The perpendicularity of the gradient on the optimal solutions only applies when small perturbations on the matrix does not make the two complex eigenvalues jump to two real ones. 
The real inner product of matrices $\nabla\left(\alpha\left(K_{N+i}\right)\right)$ and $\Delta K_{N+i}$ is calculated by

$$
\begin{aligned}
\left\langle\nabla\left(\alpha\left(K_{N+i}\right)\right), \Delta K_{N+i}\right\rangle & =\sum_{k=1}^{4}\left(\nabla \alpha\left(K_{N+i}\right)\right)_{k} \Delta\left(K_{N+i}\right)_{k} \\
& =\left\langle v_{1}, \delta \bar{p}_{m}\right\rangle+\left\langle v_{2}, \delta \bar{p}_{n}\right\rangle
\end{aligned}
$$

Thus the variation of $\alpha\left(K_{N+i}\right)$ is determined by the orthogonal projections of vectors $\delta \bar{p}_{m}$ and $\delta \bar{p}_{n}$ on the directions $v_{1}$ and $v_{2}$ respectively. Equation (16) implies that when choosing the local leaders, we should focus on those agents located near the boundaries where the optimal solutions lie, such as those two bold lines in Fig. 3.

\section{Clls Densely Distributed Over An Arbitrary CONVEX DOMAIN}

For a group of arbitrary distributed agents, Assumption 3.1 may fail to be satisfied. Instead, if we consider the convex hull for the set of CLLs, the boundary optimality may not apply and the gradient inside the polygon is not as explicit as those on the boundaries. Thus the following sequential procedures are proposed to solve Problem 2.1:

i Solve the optimization problem over a continuous domain based on the gradient bundle method [11]

$$
\begin{aligned}
& \min _{p_{m}, p_{n}} \alpha\left(K_{N+i}\right) \\
& \text { s.t. } p_{m}, p_{n} \in \operatorname{conv}\left(\left\{p_{j} \mid j \in C_{N+i}\right\}\right)
\end{aligned}
$$

where $\operatorname{Conv}(S)$ is the convex hull of the set $S$. Denote the optimal solutions by $\tilde{p}_{1}$ and $\tilde{p}_{2}$, and the corresponding cost value $\alpha\left(K_{N+i}^{\star}\right)$.

ii Find the optimal pair of local leaders $p_{m^{\star}}, p_{n^{\star}}, m^{\star}, n^{\star} \in C_{N_{i}}$ from the discrete set of the CLLs around the optimal solutions $\tilde{p}_{1}$ and $\tilde{p}_{2}$.

Note that here we do NOT bring any new reference frame to the formation system, and we do not expect the convex hull to be a rectangle. Meanwhile, the outgoing edges from agent $N+i$ is determined according to local information, and the local optimality adds up to the overall optimality. Thus the design of the minimally persistent graph for the formation system is presented in an optimal and distributed way under the constraints of (18).

In the following context, we will investigate rules that are beneficial to the selection of local leaders around the optimal points.

When $\alpha\left(K_{N+i}\right)$ corresponds to a pair of coalesce eigenvalues, finding $p_{m^{\star}}, p_{n^{\star}}$ is similar to the one in Section III. When $\alpha\left(K_{N+i}\right)$ corresponds to a simple eigenvalue, the gradient of $\alpha\left(K_{N+i}\right)$ at the optimal solutions $\tilde{p}_{1}$ and $\tilde{p}_{2}$ could be estimated by the condition number of the eigenvalues.

When there are perturbations at the optimal solution $\tilde{p}_{1}$ and $\tilde{p}_{2}$, i.e., $\delta \tilde{p}_{1}=\left(\delta \tilde{x}_{1}, \delta \tilde{y}_{1}\right)$ and $\delta \tilde{p}_{2}=\left(\delta \tilde{x}_{2}, \delta \tilde{y}_{2}\right)$, matrix $K_{N+i}$ is perturbed by

$$
\Delta K_{N+i}=\left[\begin{array}{cc}
-\delta \tilde{x}_{1} & -\delta \tilde{y}_{1} \\
\delta \tilde{x}_{2} & \delta \tilde{y}_{2}
\end{array}\right]
$$

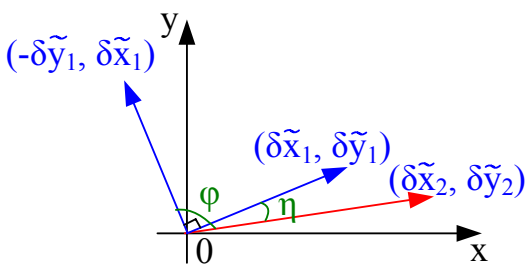

Fig. 4: Vectors under small perturbations

Lemma 4.1: Consider $K_{N+i}^{\star}$ with the largest eigenvalue $\bar{\lambda}\left(K_{N+i}^{\star}\right)$ being a simple one. Then

$$
\Delta \bar{\lambda}\left(K_{N+i}^{\star}\right)=\frac{y^{T}\left(\Delta K_{N+i}\right) x}{y^{T} x}+O\left(\left\|\Delta K_{N+i}\right\|_{2}^{2}\right)
$$

and

$\left.\left|\Delta \bar{\lambda}\left(K_{N+i}^{\star}\right)\right| \leq \operatorname{cond}\left(K_{N+i}^{\star}\right)\right)\left\|\Delta K_{N+i}\right\|_{2}+O\left(\left\|\Delta K_{N+i}\right\|_{2}^{2}\right)$

where the individual condition number of $K_{N+i}^{\star}$ is

$$
\operatorname{cond}\left(K_{N+i}^{\star}\right)=\frac{\|x\|_{2}\|y\|_{2}}{\left|y^{T} x\right|}
$$

where $x$ and $y$ are the right and left eigenvectors of $\bar{\lambda}\left(K_{N+i}^{\star}\right)$.

Proof: This is a straightforward extension of the result given in [13], p. 183.

Instead of focusing on the variation of the spectra abscissa which is difficult to track, we switch to its upper bound (21). In other words, in order to find two local leaders that minimize the spectral abscissa of $K_{N+i}+\Delta K_{N+i}$, one need to find two candidate local leaders around the optimal solutions such that the 2-norm of $\Delta K_{N+i}$ is minimized.

Theorem 4.1: Assume the optimal solutions to Problem (18) are $\tilde{p}_{1}$ and $\tilde{p}_{2}$. Let $\delta \tilde{p}_{1}$ and $\delta \tilde{p}_{2}$ be the variations on $\tilde{p}_{1}$ and $\tilde{p}_{2}$ respectively with the subtended angle $\eta$. Then it is sufficient that the optimal local leaders in Problem 2.1 are the two agents with small values on $\left|\delta \tilde{p}_{1}\right|$ and $\left|\delta \tilde{p}_{2}\right|$, and with $\eta$ being close to 90 degree.

Proof:

$$
\begin{aligned}
\Delta K_{N+i}^{T} \Delta K_{N+i} & =\left[\begin{array}{cc}
\delta \tilde{x}_{1} & \delta \tilde{x}_{2} \\
\delta \tilde{y}_{1} & \delta \tilde{y}_{2}
\end{array}\right]\left[\begin{array}{cc}
\delta \tilde{x}_{1} & \delta \tilde{x}_{2} \\
\delta \tilde{y}_{1} & \delta \tilde{y}_{2}
\end{array}\right] \\
& =\left[\begin{array}{cc}
\delta \tilde{x}_{1}^{2}+\delta \tilde{x}_{2}^{2} & \delta \tilde{x}_{1} \delta \tilde{y}_{1}+\delta \tilde{x}_{2} \delta \tilde{y}_{2} \\
\delta \tilde{y}_{1} \delta \tilde{x}_{1}+\delta \tilde{y}_{2} \delta \tilde{x}_{2} & \delta \tilde{y}_{1}^{2}+\delta \tilde{y}_{2}^{2}
\end{array}\right]
\end{aligned}
$$

If we denote $\delta d_{1}^{2}=\delta \tilde{x}_{1}^{2}+\delta \tilde{y}_{1}^{2}$ and $\delta d_{2}^{2}=\delta \tilde{x}_{2}^{2}+\delta \tilde{y}_{2}^{2}$, then

$$
\begin{aligned}
& \bar{\lambda}\left(\Delta K_{N+i}^{T} \Delta K_{N+i}\right) \\
& =\frac{1}{2}\left(\delta d_{1}^{2}+\delta d_{2}^{2} \pm \sqrt{\left(\delta d_{1}^{2}+\delta d_{2}^{2}\right)^{2}-4\left(\delta \tilde{x}_{2} \delta \tilde{y}_{1}-\delta \tilde{x}_{1} \delta \tilde{y}_{2}\right)^{2}}\right)
\end{aligned}
$$

The expression $\delta \tilde{x}_{1} \delta \tilde{y}_{2}-\delta \tilde{x}_{2} \delta \tilde{y}_{1}$ can be considered as an inner product of two vectors $\left(\delta \tilde{x}_{2}, \delta \tilde{y}_{2}\right)$ and $\delta \bar{p}_{1}^{\perp}=$ $\left(-\delta \tilde{y}_{1}, \delta \tilde{x}_{1}\right)$ where " $\delta \bar{p}_{1}^{\perp}$ " is a vector that is obtained by rotating vector $\delta \tilde{p}_{1}=\left(\delta \tilde{x}_{1}, \delta \tilde{y}_{1}\right) 90$ degrees counter clockwise, as shown in Fig. 4. Assume the angle between $\delta \bar{p}_{1}^{\perp}$ 


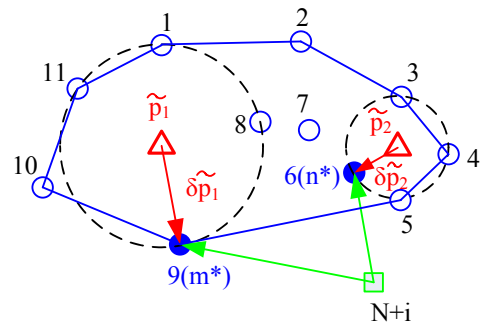

Fig. 5: Agent 6 and agent 9 is one pair of optimal local leaders to agent $N+i$

and $\delta \tilde{p}_{2}$ is $\varphi$, and the angle between $\delta \tilde{p}_{1}$ and $\delta \tilde{p}_{2}$ is $\eta$ that satisfy $\sin ^{2} \eta=\cos ^{2} \varphi$. Thus

$$
\left(\delta \tilde{x}_{2} \delta \tilde{y}_{1}-\delta \tilde{x}_{1} \delta \tilde{y}_{2}\right)^{2}=\delta d_{1}^{2} \delta d_{2}^{2} \cos ^{2} \varphi=\delta d_{1}^{2} \delta d_{2}^{2} \sin ^{2} \eta
$$

which further yields

$$
\left\|\Delta K_{N+i}\right\|_{2}^{2}=\frac{1}{2}\left(\delta d_{1}^{2}+\delta d_{2}^{2}+\sqrt{\left(\delta d_{1}^{2}+\delta d_{2}^{2}\right)^{2}-4 \delta d_{1}^{2} \delta d_{2}^{2} \sin ^{2} \eta}\right)
$$

According to (26), $\left\|\Delta K_{N+i}\right\|_{2}$ is determined by the angle $\eta$ and by the distance between the CLLs and the optimal positions. This indicates that when choosing the pairs of CLLs, we should focus on those pairs of agents that both locate close to $\tilde{p}_{1}, \tilde{p}_{2}$, i.e., have small $\delta d_{1}$ and $\delta d_{2}$, and form an angle around 90 degree, i.e., $\sin \eta$ is as large as possible.

Remark 4.1: This theorem guides us to find the two local leaders with the best chance of being the optimal pair. In order to find its local leaders, agent $i$ is required to generate a convex hull according to the positions of agents within its sensing area rather than the positions of all the other agents in the formation system. Information in the system is distributed in terms that each agent can make the best choice of its local leaders autonomously using only local information without modifying the existing communication topology.

We consider a special situation when most of the CLLs are either on the circle centered at the optimal position $\tilde{p}_{1}$ or $\tilde{p}_{2}$, as in Fig. 5. The two red triangles is the optimal solution to (18). In such a case, $\delta d_{m}$ and $\delta d_{n}$ are fixed. As indicated by (26), the optimal local leaders should appear pairwise from those two circles, and thus $\eta$ is the crucial variable to $\left\|\Delta K_{N+i}\right\|_{2}$. In order to minimize its value, $\sin ^{2} \eta$ should be as large as possible, and thus $\eta$ should be around 90 degrees. The two red arrows in the figure are the ones that meet the above demands. So node 9 and node 6 is the optimal pair of local leaders to node $N+i$. On the contrary, if we choose node 5 instead of node 6 , it would yield $\sin \eta \rightarrow 0$, thus $\left\|\Delta K_{N+i}\right\|_{2}^{2}$ would be larger than the case with node 9 and node 6 .

\section{CONCLUSION}

In this paper, the optimal local leader selection of a LFFMP formation system is discussed. The generation of the optimal minimally persistent graph is carried out in a distributed and subsequential way. At each step, only one unsettled agent is considered and is assigned with two optimal local leaders using only information within its sensing range. When the CLLs are densely distributed over a rectangular area, the optimal solution exhibits boundary optimality. Further more, when agents are uniformly distributed over an arbitrary area, the optimal pair of local leaders are selected based on the matrix perturbation theory.

From the overall system perspective, the convergence time of agent $i$ is determined by its local convergence rate towards $\bar{p}_{i}$ and the convergence rate of its two local leaders $m$ and $n$. Thus as long as agent $m$ or agent $n$ is moving, the stable position of agent $i$ is changing as well. Ideally, having the $M$ unsettled agents looking at the leader and the first follower might be the best choice because they converge to the final stable position much faster than the other followers. However this is not applicable due to limited communication range and the lack of robustness. For the future work, when extending the current objective function concerning the local convergence rate to the one corresponding to the global convergence rate, it would be important to figure out how to explicitly measure the local convergence rate and the priority of the local leaders.

\section{REFERENCES}

[1] R. Abel, S. Dasgupta, and J. Kuhl, "The relation between redundancy and convergence rate in distributed multi-agent formation control," in Proceedings of the 47th IEEE Conference on Decision and Control, 9-11 2008, pp. $3977-3982$.

[2] J. Fax and R. Murray, "Information flow and cooperative control of vehicle formations," IEEE Transactions on Automatic Control, vol. 49, no. 9, pp. 1465-1476, 2004.

[3] G. Zhu and J. Hu, "Stiffness matrix and quantitative measure of formation rigidity," in Proceeding of the 48th IEEE Conference held jointly with the 2009 28th Chinese Control Conf Decision and Control CDC/CCC 2009, 2009, pp. 3057-3062.

[4] Y. Kim, G. Zhu, and J. Hu, "Optimizaing formation rigidity under connectivity constraints," in Proceedings of 49th IEEE International Conference on Decision and Control, Atlanta, GA, Dec. 2010, pp. $6590-6595$.

[5] I. Shames, A. M. H. Teixeira, H. Sandberg, and K. H. Johansson, "Distributed Leader Selection without Direct Inter-agent Communication," in Proceedings of the IFAC Workshop on Distributed Estimation and Control in Networked Systems, Annecy, France, 2010, pp. 221 226.

[6] S. Kar, S. Aldosari, and J. Moura, "Topology for distributed inference on graphs," IEEE Transactions on Signal Processing, vol. 56, no. 6, pp. $2609-2613$, jun. 2008.

[7] R. Olfati-Saber, "Ultrafast consensus in small-world networks," in Proceedings of the 2005 American Control Conference, jun. 2005, pp. $2371-2378$ vol. 4.

[8] S. Patterson and B. Bamieh, "Leader selection for optimal network coherence," in 49th IEEE Conference on Decision and Control, pp. 2692-2697.

[9] J. M. Hendrickx, B. Fidan, C. Yu, B. D. O. Anderson, and V. D. Blondel, "Formation reorganization by primitive operations on directed graphs," vol. 53, no. 4, pp. 968-979, 2008.

[10] C. Yu, B. D. O. Anderson, S. Dasgupta, and B. Fidan, "Control of minimally persistent formations in the plane," SIAM Journal on Control and Optimization, vol. 48, no. 1, pp. 206-233, 2009. [Online]. Available: http://link.aip.org/link/?SJC/48/206/1

[11] J. V. Burke, A. S. Lewis, and M. L. Overton, "Two numerical methods for optimizing matrix stability," Linear Algebra and its Applications, vol. 351-352, pp. 117 - 145, 2002.

[12] R. A. Horn and C. R. Johnson, Matrix Analysis. Cambridge University Press, 1985.

[13] G. W. Stewart and J. guang Sun, Matrix Perturbation Theory. Academic Press, 1990. 